\title{
International Journal of Medicine
}

\section{Differential diagnosis of myelopathies}

\author{
Raja Sawaya, MD*, Zahi Nachef MD \\ Department of Neurology, American University of Beirut Medical Center, Beirut, Lebanon. \\ *Corresponding author E-mail: rs01@aub.edu.lb
}

\begin{abstract}
We present a review on myelopathies. We introduce each category of etiologies by a case study, followed by a short review of the different causes of myelopathies in this category. We elaborate on clinical presentation, laboratory workup, imaging and treatment. This extensive review elaborates on myelopathies caused by demyelinating diseases, infectious agents, connective tissue diseases and vasculopathies, neoplasms, toxic substances, metabolic derangements and the idiopathic myelopathies. Interesting images are incorporated in this review.
\end{abstract}

Keywords: myelopathies, spinal cord, demyelinating, infectious, neoplasm, idiopathic.

\section{Introduction}

Diseases affecting the spinal cord are not rare in neurology and medicine. There are many causes for pathologies of the spinal cord. Some are intrinsic disease of the neuronal structures and tracts of the spinal cord, and others are systemic illnesses that affect the morphology and physiology of the spinal cord (table 1). Myelopathies have different clinical presentations even though the tissues affected are the same. The history and physical findings are of great importance in setting the stage for further work up. MRI imaging of the affected segments usually confirm the diagnosis as there are specific images for the different etiologies. Evoked potential studies are important in defining the extent of functional disability of the sensory and motor tracts of the cord. Serum and CSF studies help in defining the etiologies of the causative agents in many diseases. Treatment has become available for most if not all the causes of myelopathies as detailed below.

This is a medical record and literature review. No approval needed from local authorities.

\section{Demyelinating myelopathies:}

\subsection{Case Report}

This is a 35 year old female who presented with four days history of progressive weakness of the left lower extremity with no sensory symptoms. Neurological examination revealed weakness of proximal and distal muscles of the left leg. Normal sensory examination for all modalities. Hyperreflexia in all four limbs. Abnormal tandem walking. Abnormal Romberg. Normal cranial nerves. She gave history of acute diplopia with spontaneous resolution seven years ago. MRI of the brain and cervical spine revealed multiple hyper intense $\mathrm{T} 2$ lesions in the white matter in the brain and spinal cord (fig 1). Abnormal visual evoked potentials. Elevated CSF IgG with presence of oligoclonal bands and absent CSF white blood cells.
Table 1: The different etiologies of myelopathies

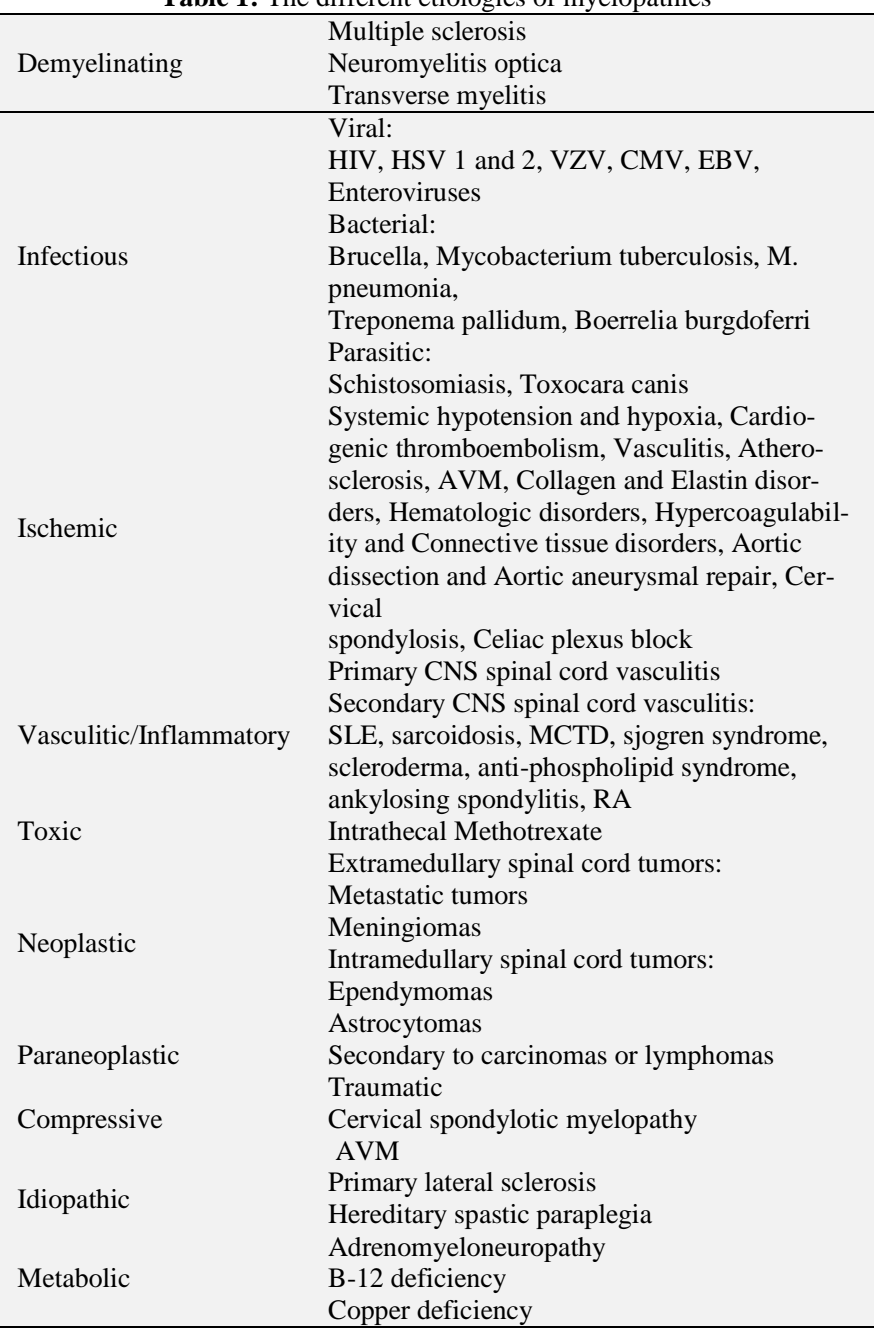




\subsection{Diagnosis: demyelinating myelopathy}

\subsubsection{Discussion}

Demyelinating myelopathies usually present as partial or complete transverse myelitis. It affects individuals of all ages with a bimodal peak of 10-19 and 30-39 years. No sex or familial predisposition. It is characterized by acute or subacute presentation of symptoms and signs of neurological dysfunction in motor, sensory, and autonomic systems. Weakness is usually rapidly progressive characterized by ascending paraparesis. Pyramidal signs appear by the second week of the illness. A sensory level can be documented in most cases. Autonomic symptoms are mainly urinary urgency, bowel or bladder incontinence, retention, and sexual dysfunction. Seventy five to $90 \%$ of transverse myelitis is monophasic while the rest are multiphasic. Hyperintense lesions on T2 weighted MRI images appear in the white matter, less than 2 spinal cord segments favor multiple sclerosis, while more than 3 segments favor neuromyelitis optica.

CSF findings include mild pleocytosis, elevated protein level, elevated IgG content with presence of oligoclonal banding. The presence of protein 14-3-3 in the CSF is a marker of poor outcome and the levels of interleukin 6 are proportional to disability in subjects prior to treatment. Demyelinating myelopathies are treated primarily by high dose intravenous steroids especially in the acute phase. Plasma exchange and/or intravenous immunoglobulin are treatments used in patients with severe motor or autonomic dysfunction who fail to improve after pulse steroid therapy. Predictors of good response to plasma exchange include early treatment (less than 20 days from symptom onset), and a clinically incomplete lesion (Cordonnier et al.2003, Bashir et al.2000, Kaplin et al.2005).

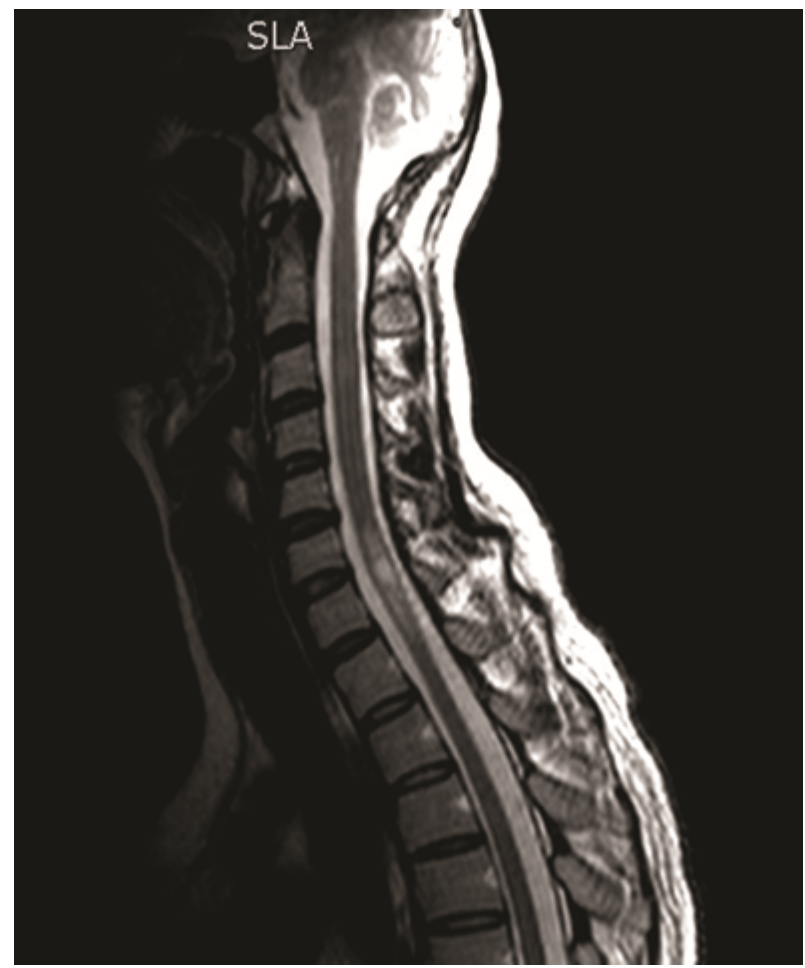

Fig. 1: MRI of the cervical spine, sagital view, T2 weighted-image revealing a hyperintense intraspinal lesion with edema compatible with a demyelinating lesion.

\section{Infectious myelopathies:}

\subsection{Case Report:}

This is a 30 year old man who presented with six months history of low back pain and two months history of progressive weakness in the left lower extremity associated with urinary retention and impotence. No sensory symptoms. Neurological examination revealed no focal motor weakness. Generalized hyperreflexia. Bilateral clonus. Babinski on the left side. Normal sensory examination. Normal cranial nerves. No cerebellar signs and normal gait. MRI of the brain was normal. MRI of the thoracic spine revealed a fusiform intramedullary lesion at D5 with edema of the cord at that level. Hypersignal intensity on T2 and FLAIR and postGadolinium enhancement. Normal visual and brainstem evoked responses. Delayed sensory and motor evoked responses. CSF studies revealed isolated hypereiosinophilia with normal WBC count, glucose, and IgG level. Oligoclonal bands were present. CSF gram stain and cultures were negative, Toxocara canis antibodies were positive by ELISA and Western blot.

\subsection{Diagnosis: Toxocara canis Myelitis}

\subsubsection{Discussion}

Infections of the spinal cord are usually of viral etiology with good prognosis and spontaneous recovery. The most common viruses causing myelopathies are herpes simplex 1 and 2, varicella zoster, cytomegalo virus, epstain barr virus and enteroviruses. The CSF usually reveals lymphocytic pleocytosis with normal protein and glucose level with high IgG and positive PCR for the culprit virus. On the other hand AIDS myelopathy caused by the HIV virus causes vacuolar degeneration of the spinal cord resulting in progressive neurological deficit and eventual death. Bacterial infections causing myelopathies are usually progressive and require antibacterial treatment. Brucellosis, mycobacterial tuberculosis, mycoplasma pneumonia, treponema pallidum, and borrelia burgdoferri are the most common agents encountered. CSF studies usually reveal polymorphocytic pleocytosis, and low sugar level. Treatment depends on identifying the agent by stain and cultures, antigen detection or PCR identification. Parasitic infiltration of the spinal cord is rare and usually includes shistosomiasis or toxocaraesis. These are usually diagnosed by CSF eosinophelia, and antigen detection of the culprit parasite. Treatment is by oral antiparasitic agents for 3-6 months with good outcome (Kaplin et al.2005, Meurs et al.2004, Staudinger et al.2000, Di Rocco 1999, Orme et al.2007, Kincaid et al. 2006, Fux et al.2003).

Epidural abscess also cause myelopathies, either by mechanical compression or as a results of vascular occlusion by septic thrombophlebitis. Epidural abscesses can result from hematogenous dissemination or local extension from vertebral or disc infections. The predominant manifestations of epidural abscesses, in addition to the symptoms of cord compression, are back pain and fever. Leukocytosis, elevated ESR and CRP occur in $70-100 \%$ of cases. Bacteremia is detected in $60 \%$ especially in staphylococcus aurous infections. CSF studies reveal elevated protein, low sugar and polymorphonuclear pleocytosis. CSF culture usually defines the etiologic agent and if this fails then a CT-guided aspirate of the abscess is necessary for diagnosis. Treatment is a combination of intravenous antimicrobial therapy and surgical excision of the abscess. Prognosis depends primarily on the degree of neurological deficit upon diagnosis (Darouiche 2006).

\section{Ischemic myelopathies}

\subsection{Case Report:}

This is a 50 year old female previously healthy who presented with acute motor paralysis of the lower extremities. She had complete absence of sensation below D10, urinary retention and lax anal tone. MRI of the spinal cord revealed a hyperintense, nonenhancing, lesion on T2 weighted images in the spinal cord at D10 level compatible with an ischemic lesion. Spinal angiography revealed occlusion of the AdamKiwich artery. The patient was started on intravenous heparin with stabilization of the neurological deterioration. Blood studies including vasculitis studies were 
normal. MRI brain was normal and CSF studies were normal Hypercoagulable profile was assessed and the patient was found to have a homozygous mutation C677T of the methylenetetrahydrofolate reductase gene.

\subsection{Diagnosis: ischemic myelopathy.}

\subsubsection{Discussion:}

Infarction of the spinal cord is rare. The most common cause is embolisation or thrombosis of the anterior spinal artery infarcting the anterior two thirds of the cord. These patients present with abrupt flaccid paralysis of the lower extremities with areflexia, loss of spinothalamic perception of pain and temperature, and autonomic deficits leading to sphincter flaccidity, atonic bladder, and paralytic ileus. Transient severe radicular or back pain usually heralds these deficits. Spasticity will ensue eventually with exaggerated deep tendon reflexes, Babinski signs and clonus.

The most common etiologies of spinal cord infarction are systemic hypotension and hypoxia, cardiogenic thromboembolism, vasculitis, atherosclerosis, AVM, collagen and elastin disorders, hematologic disorders, hypercoagulability and connective tissue disorders, aortic dissection and aortic aneurysmal repair, cervical spondylosis, celiac plexus block. Diagnosis of spinal cord infarction is made by MRI imaging of the cord which shows hypodense lesion on T2 sequences and abnormal diffusion-perfusion scanning (fig 2). Segmental spinal angiography is the imaging of choice but is not always successful because of difficulty in accessing the spinal arteries. Treatment involves permanent anticoagulation, addressing the underlying etiology and supportive care to avoid secondary complications. Prognosis for recovery is usually grave despite early treatment ((Cheshire et al.1996, Faig et al.1998, Mahfouz et al.2006).

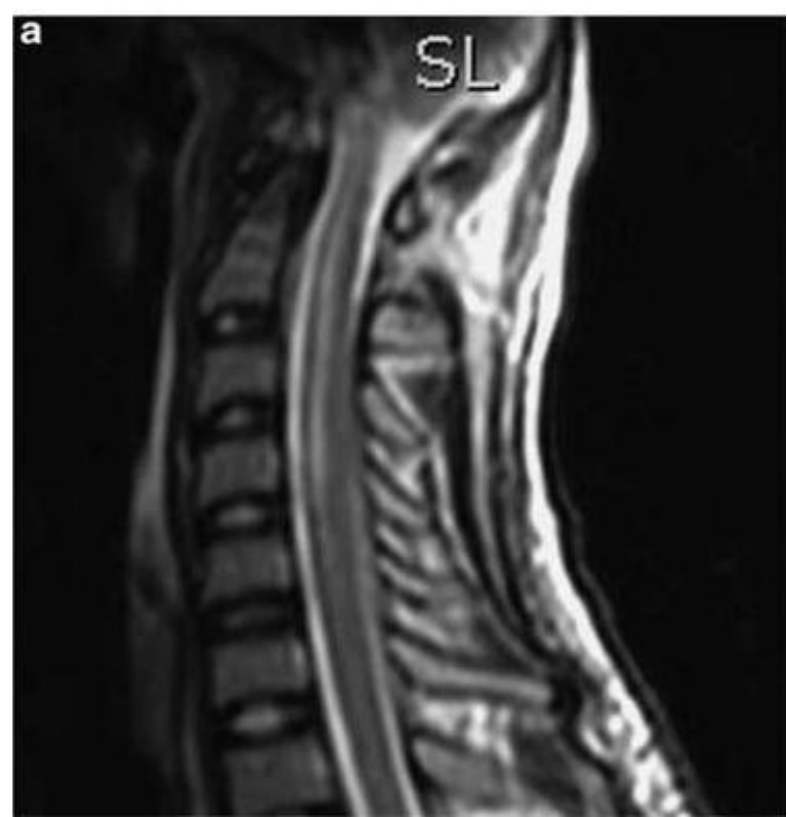

Fig. 2: MRI of the cervical spinal cord, sagital view, T2-weighted image revealing a hyperintense lesion of the anterior cord extending from $\mathrm{C} 2$ to C5 vertebra suggestive of an ischemic lesion.

\section{Vasculitic/inflammatory myelopathies.}

\subsection{Case report:}

This is a 46 year old female who presented with ascending progressive numbness and parasthesias involving the four extremities. Motor power was preserved. Sphincter abnormalities ensued. The patient had type I diabetes mellitus and hypothyroidism. Neurological examination revealed preserved motor power in the lower extremities. Decreased sensation for pain and temperature in the left leg below the knee. Decreased position and vibratory sense in the legs. Hyperreflexia of the patellar reflex. Absent Achilles reflexes bilaterally. Normal gait. No Babinski signs. No cerebellar signs. Blood studies were normal except for normocytic anemia, elevated $\mathrm{HbA} 1 \mathrm{C}$, positive ANA (anti-ds DNA was normal). CSF studies were normal, including infectious workup as well as negative oligoclonal bands and ACE levels. Evoked potential studies were normal. MRI brain was normal; MRI spine revealed extensive edema involving the cervical spinal cord from $\mathrm{C} 1$ to $\mathrm{C} 7$ associated with nodular enhancement in the posterior aspect (fig 3). Biopsy of the cervical cord revealed the presence of inflammatory cells composed of mature lymphocytes and lipid-laden macrophages, no malignant cells seen. The patient was treated with IV pulse steroid therapy followed by oral steroids and immunotherapy. Follow up examination revealed resolution of the symptoms and signs, and normalization of the MRI image.

\subsection{Diagnosis: Vasculitic myelopathy.}

\subsubsection{Discussion:}

Vasculitic myelopathy can be either secondary to primary CNS vasculitis or part of a systemic connective tissue disease. In primary CNS vasculitis, spinal cord involvement is much less frequent than cerebral involvement. The pathology is usually necrotizing or granulomatous. Secondary vasculitic myelopathy is seen in SLE, sarcoidosis, mixed connective tissue disease, Sjogren's syndrome, scleroderma, antiphospholipid antibody syndrome, ankylosing spondylitis, and rheumatoid arthritis. The most common vasculitis causing myelopathy are SLE and sarcoidosis. In sarcoidosis the myelopathy is the presenting symptom in $50 \%$ of patients. Diagnosis is made by MRI imaging revealing swelling of the cord with gadolinium enhancement. CSF studies reveal elevated protein, lymphocytic pleocytosis and decreased glucose level. IgG level is not usually elevated, CSF ACE level is elevated in 50\% of cases and is useful in monitoring disease activity. Level of CSF ACE does not correlate with serum level. CSF lysosome and beta2microglobulin levels are elevated but not specific. Serum ACE level is elevated in less than $40 \%$ of cases. Treatment for the myelopathy follows the same protocol of systemic sarcoidosis, mainly high dose steroid treatment and immunosuppressive therapy, with around $60 \%$ improvement rate (Saleh et al. 2006, Kumar et al. 2004, Krishnan et al.2004, Mok et al.1995, Anantharaju et al.2003, Torabi et al.2004, de Seze et al.2005, Harzheim et al.2004).

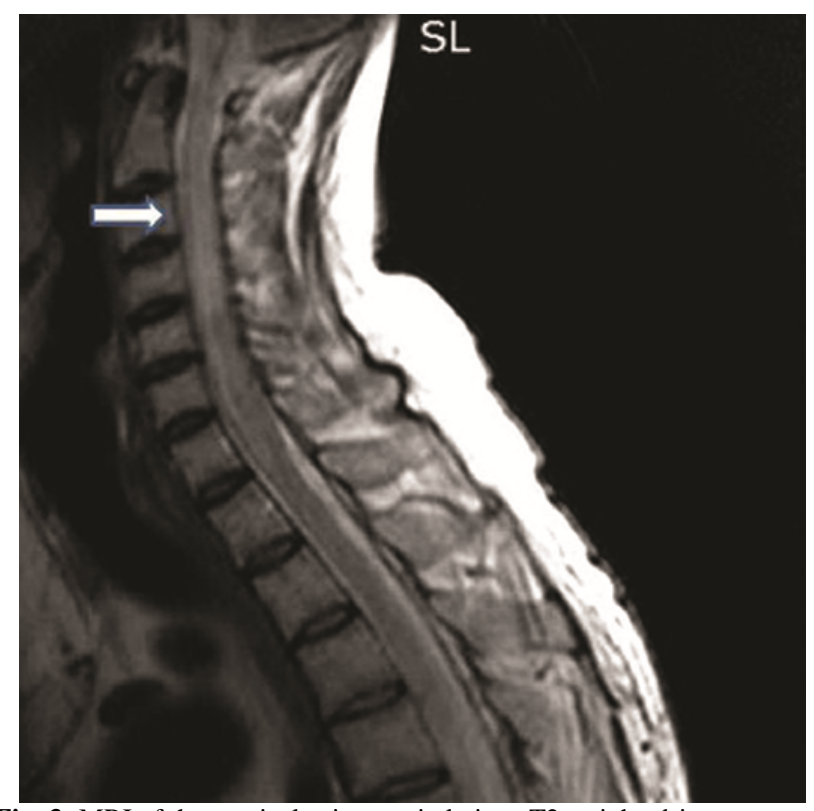

Fig. 3: MRI of the cervical spine, sagital view, T2 weighted-image revealing swelling of the cervical cord with no masses. 


\section{Toxic myelopathy}

\subsection{Case report}

This is a 45 year old man diagnosed with Burkett's lymphoma who was treated with rituximab and hyper CVAD regimen along with standard prophylaxis for CSF lymphoma including intrathecal methotrexate and cytarabine. After the second intrathecal methotrexate injection the patient developed progressive ascending pain, parasthesias, and motor weakness up to and involving the upper cervical cord. Sphincter dysfunction ensued. MRI spine revealed diffuse enhancing lesion of the white and gray matter throughout the cervical and dorsal cord. CSF studies revealed normal cell count, elevated protein and $\mathrm{IgG}$ index, with no malignant cells. EMG and evoked potentials studies revealed demyelinating motor polyneuropathy with abnormal sensory and motor tract functions. Serum anti-Hu, anti-Yo, and anti-CRMP 5 were negative. CT abdomen revealed regression of the lymphoma The patient received high dose IV steroid and IVIG therapy and intrathecal steroid therapy with no improvement of his symptoms.

\subsection{Diagnosis: toxic myelopathy}

\subsubsection{Discussion}

Intrathecal methotrexate is a treatment which has been reported to produce myelopathy and rarely radiculopathy. The myelopathy occurs in less than $3 \%$ of patients and occur early after the injection. It is usually self-limiting or reversible and can occur after a single or multiple injections. Neuropathological findings in these cases include myelin necrosis, microvacuolisation and scattered axonal swelling in the spinal cord. These pathological changes are usually limited to the lateral parts of the cord and can involve the whole length of the spinal cord. The pathology usually involves the anterior motor roots with preservation of the sensory roots and fibers. The pathological mechanism is unclear and occurs with or without the use of preservatives in the injected fluid. Local depletion of folate secondary to methotrexate injection has been suggested. Intrathecal methotrexate may cause a breakdown of the blood-brain barrier of the nerve roots of the cauda equina where it is injected. This breakdown may facilitate the direct toxic effect of the drug or may also provoke an immune response. Treatment of such a condition involves primarily the cessation of the intrathecal treatment, followed by a trial of high dose intravenous and low dose intrathecal steroids as early as diagnosis. Nevertheless, the prognosis is usually independent of the therapy. Patients may stabilize with permanent deficit or recover spontaneously. There exist few reports of progressive deterioration inspite of treatment (Pascual et al.2008, Bay et al.2005, Lee et al.2008, Clark et al.1982)

\section{Neoplastic myelopathy:}

\subsection{Case report:}

This a 35 year old man who presented with 10 days history of neck pain radiating to both upper extremities. Neurological examination revealed normal motor power, sensory function and deep tendon reflexes in the upper extremities. On the other hand examination revealed mild spasticity in the lower extremities, hyperreflexia of the reflexes in the legs and bilateral Babinski signs. MRI of the cervical cord revealed a well circumscribed oval-shaped mass compressing the spinal cord at C4 level. It shows increased signal intensity on $\mathrm{T} 2$ and partial faint contrast enhancement (fig 4). Normal posterior tibial somatosensory evoked potentials. Surgical biopsy revealed a low grade astrocytoma.

\subsection{Diagnosis: Malignant myelopathy}

\subsubsection{Discussion}

Tumors of the spinal cord can be of benign or malignant type and can injure the cord either by external compression or intramedullary growth.

Symptoms of spinal cord tumor usually are progressive weakness below the level of the lesion with accompanying sensory loss and bladder dysfunction. Pain at the site of involvement is typical. Progression to paraplegia can occur abruptly, as a result of vascular compression.

Extramedullary spinal cord tumors are usually meningiomas or metastatic tumors.

Intramedullary spinal cord tumors are usually ependymomas and astrocytomas.

Diagnosis of spinal cord tumors starts with the clinical suspicion and is confirmed by MRI imaging. The MRI typically shows masses which may or may not enhance depending on their pathological types. Meningiomas are usually tailed to the dura and enhance homogenously, while ependymomas and astrocytomas do not enhance heavily. The definite diagnosis is usually by surgical biopsy or excision. Treatment starts by high dose intravenous steroids to decrease spinal cord edema. Benign tumors are treated by total surgical excision while malignant tumors are treated by radiotherapy (Shrivastava et al.2005, Sevick et al.1999, HelwegLarsen et al.1999).

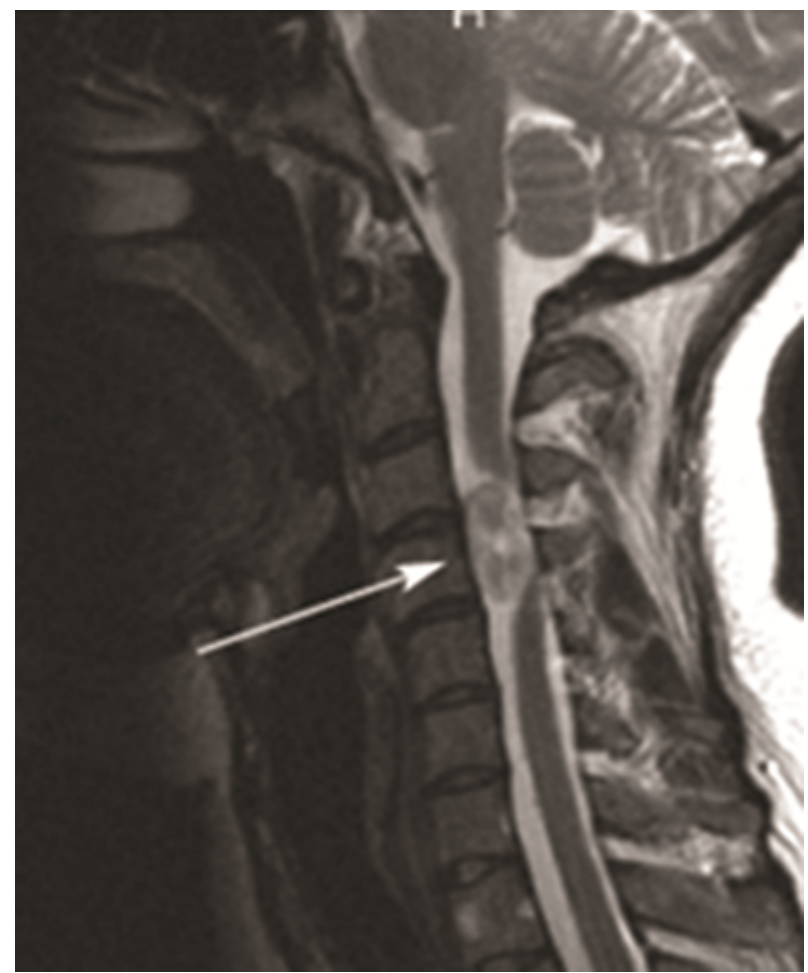

Fig. 4: MRI of the cervical cord, sagital view, T2 weighted-image revealing an extra-axial tumor at the $\mathrm{C}-4$ level compressing the cord compatible with meningiomas.

\section{Paraneoplastic myelopathy:}

\subsection{Case report:}

A 75 year old man presented with a history of progressive weakness of the lower extremities associated with decreased sensations from the umbilicus downwards and urinary retention. Past medical history is negative except for the history of chronic heavy smoking. The symptoms progressed over 3-4 weeks. Physical examination revealed spastic weakness of the lower extremities. Hyperreflexia of the lower extremity DTRs. Bilateral Babinsky signs. Full urinary bladder on palpation. Lax anal sphincter. Sensory level at D-10. Blood studies were normal. MRI spine re- 
vealed diffuse hyperintense lesion along the dorsal cord form D7 D10 with patchy enhancement. Chest X-ray and CT chest revealed a large tumor in the right upper lobe. Surgical excision revealed an adenocarcinoma. The patient received chemotherapy and radiotherapy over 9 months. During this period the neurologic deficits improved significantly. He regained power in his legs and a balanced gait. Sphincter functions improved inspite of requiring sporadic catheterization. Sensory level disappeared.

\subsection{Diagnosis: paraneoplastic myelopathy}

\subsubsection{Discussion}

Degeneration of central and peripheral nervous system has also been considered as a paraneoplastic phenomenon. Paraneoplastic neurological symptoms are classified as definite or possible depending on the clinical syndrome defined, the response of the syndrome to the treatment of the cancer, the presence or absence of well-characterized onco-neural antibodies which are anti- $\mathrm{Hu}$, anti-Yo, anti-CV2 , anti-Ri, anti-MA 2 and anti- amphyphisin. Paraneoplastic syndromes are usually associated with lymphoproliferative disorders and can present as motor neuron disease, peripheral neuropathy, neuromyotonia, cerebellar degeneration, and inflammatory myelopathies. Paraneoplastic myelopathy is observed with carcinoma or lymphoma and often called "subacute necrotizing paraneoplastic myelopathy". CSF findings are usually normal or show mild elevated protein and mild pleocytosis. No malignant cells are present in the CSF and no oligoclonal bands. Autopsy studies reveal myelin and axonal degeneration as well as vacuolization of the spinal cord involving white and gray matter not confined to particular tracts. No evidence for tumor infiltration or vasculitic process in the affected tissues (Toothaker et al.2009, Darnell et al. 2003, Darnell 2004).

\section{Compressive myelopathy:}

\subsection{Case report:}

This is a 78 year old man previously healthy who presented with several years of slowly progressive numbness and pain symmetrically over both forearms and hands. The patient complained also of bilateral neck and shoulder pain and rigidity in the movement of the neck. Physical examination revealed severe weakness in the intrinsic hand muscle bilaterally with atrophy mostly pronounced in the hands. Decreased sensation in all the sensory dermatomes. Areflexia in the DTR of the upper extremities. Hyperreflexia in the patellar DTR bilaterally. Bilateral Babinski and Hoffman signs. Rigidity in the neck with lancinating pains in the upper extremities upon neck extension. Partial sphincter dysfunction reported. MRI of the cervical cord revealed severe multisegmental stenosis secondary to protruding discs and ligamental hypertrophy (fig 5). Hyperintense lesion in the center of the cord seen on T2 weighted images. EMG examination revealed severe chronic neurogenic changes with ongoing denervation in the C5-C6-C7 and $\mathrm{C} 8$ roots bilaterally. Sensory evoked potentials from the posterior tibial nerves were delayed.

\subsection{Diagnosis: compressive myelopathy.}

\subsubsection{Discussion:}

Cervical spondylotic myelopathy is the most common cause of spinal dysfunction in the elderly and the most common cause of non-traumatic spastic paraparesis and quadreparesis. It is the result of disc degeneration and ligamental hypertrophy and osephication. The myelopathy occurs as a result of static mechanical factors, dynamic mechanical factors and spinal cord ischemia. The hallmark symptoms are gait abnormalities, weakness and stiffness of the legs, as well as loss of manual dexterity, and numbness in the hands. MRI is the radiographic study of choice. It reveals the de- gree of bony involvement, ligamental hypertrophy, narrowing of the foramina, as well as the extent of spinal cord ischemia. It is also important to evaluate the number of levels involved to direct the surgical planning. It also is important to rule out other etiologies of spinal cord dysfunction. Electrophysiological studies are also important in defining the level and degree of root pathology as well as the involvement of the sensory and motor tracts by sensory and motor evoked potential analysis. Treatment of such a condition depends on the degree of neurologic deterioration and the patient's general medical condition. In mild cases of root involvement treatment can be conservative including cervical traction and non-steroidal anti-inflammatory treatment. Occasionally intra-root steroid infiltration can be used for pain relief, especially in patients with high risk for surgery. In severe compressive myelopathies, especially when there is clinical or radiological evidence for spinal cord infarction, the treatment of choice is surgical decompression and stabilization in case of unstable vertebral column (Baron et al.2007, McCormick et al.2003).

Rarely compressive myelopathies can be secondary to vascular malformations or arteriovenous fistulas (70\% of vascular malformations) compressing the cord by their sizes and intravascular pressures. These lesions are diagnosed primarily by their MRI images which show masses with vascular loops and flow void indicating vascular structure. The pathophysiological mechanism for the myelopathy is either by compression of neural tissue or by ischemia due to steal phenomena. Spinal angiography is necessary for diagnosis and evaluation of the presence of solitary feeding vessels which can promote treatment by embolisation. If embolisation is not possible or fails to interrupt blood flow through the AVM or fistula then microsurgical shunt interruption is an alternative (Koch et al.2006, Aghakhani et al.2008, Ishida et al.1989).

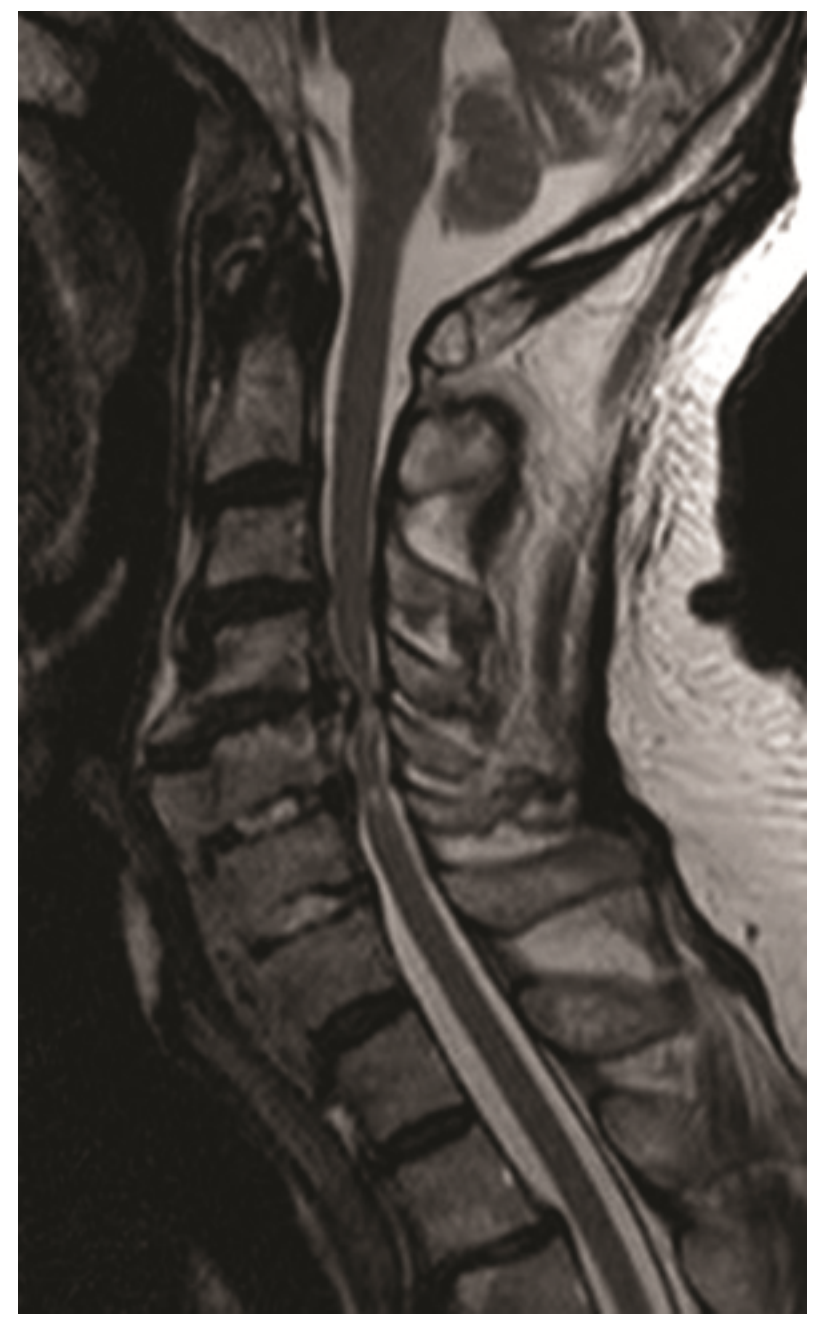

Fig. 5: MRI, cervical spine, sagital view, T2 weighted-image revealing multilevel segmental spinal stenosis with cord compression and infarction. 


\section{Metabolic myelopathy}

\subsection{Case report}

This is a 15 year old man who presented with mild gait instability and tendency to fall. Physical examination revealed mild spasticity of the lower extremities, hyperreflexia of the four limbs. No Babinski signs, no sensory dysfunction, no sphincter disturbances. Patient gave history of adrenal insufficiency since early childhood. Patient's brother has X-linked adreno-leukodystrophy. MRI of brain and spinal cord were normal. EMG revealed mild sensory motor polyneuropathy affecting the upper and lower extremities. Posterior tibial sensory evoked potentials were slightly delayed Motor evoked potentials and central motor delay were increased. Blood studies revealed evidence for adrenal insufficiency. Very long chain fatty acids VLCFA (C: 24 and 26) were elevated.

\subsection{Diagnosis: adrenomyeloneuropathy}

\subsubsection{Discussion}

Metabolic myelopathies can be hereditary or acquired. X-linked adrenomyeloneuropathy is a hereditary myelopathy secondary to VLCFA accumulation in the spinal tissue due to a defect in peroxisome betaoxidation associated with mutations in ABCD1 gene. It presents in adults as a slowly progressive paraparesis combined with sensory disturbances. The principle abnormality is a noninflammatory distal axonopathy that involves the dorsal columns, and corticospinal tracts in the lower thoracic and lumbar regions. The peripheral nerves are also involved in most patients. Diagnosis is made by measurement of plasma VLCFA which is elevated in these patients. The MRI of the spinal cord in these patients is normal. Treatment is based on lowering levels of VLCFA by decreasing intake of unsaturated fats as well as replacement of the dietary fat by Lorenzo's oil (4:1 mixture of glycerol trioleate and glycerol trierucate (Moser et al.2007,

Sawaya 2000)

Acquired metabolic myelopathies include B-12 and copper deficiencies. Vitamin B12 deficiency causes subacute degeneration of the spinal cord. The most prominent clinical and electrophysiological findings are dysfunctions of the posterior columns diagnosed by abnormally delayed or absent posterior tibial somatosensory evoked potentials associated with a low serum B12 level and no MRI evidence for other spinal cord disease. Low serum methyltetrahydrofolate and homocysteine levels are more sensitive indicators of this entity than serum B12 level. MRI sometimes reveals demyelination of the posterior columns. Peripheral nerve involvement, if present, is mostly axonal and sensory. Treatment consists of vitamin B12 replacement intramuscularly and results in good clinical recovery especially if started early in the course of the deficiency (Hemmer et al.1998).

\section{Idiopathic myelopathy}

\subsection{Case report}

This is a 57 year old man previously healthy on no medications who presented with two years history of slowly progressive dysarthria, dysphagia and gait abnormalities associated with cramps in the lower extremities and tendency to falls. Neurological examination revealed normal muscle volume in arms and legs, normal sensory examination, mild spasticity in the legs with hyperreflexia of the deep tendon reflexes in the four limbs and a lively jaw jerk. No Babinski or Hoffman signs or spastic gait. Normal cranial nerves. No cerebellar signs. Normal sphincter tone. MRI of brain and spinal cord were normal. Neurography and needle EMG examination of the upper and lower extremities were normal. Motor evoked potentials revealed evidence for pathology of the descending cortico-spinal tracts to the cervical and lumbar regions.

\subsection{Diagnosis: primary lateral sclerosis}

\subsubsection{Discussion}

Degenerative myelopathies can be hereditary or acquired, stable or slowly progressive with no known etiology at present. Primary lateral sclerosis is a disease of the descending motor tracts which presents with spasticity of the bulbar and spinal nerves and which progresses very slowly over decades. It presents with spasticity and hyperreflexia involving the muscles of the upper and lower extremities with or without Babinski signs. Fasciculations are sparse. Sensory examination and sphincter functions are preserved. Gait is spastic. Radiography of the brain and spinal cord are normal. Diagnosis is made by electrophysiological studies which reveal normal motor and sensory nerves and normal EMG examination of the proximal and distal muscles with sparse fibrillations and fasciculations if any. Motor units have normal configuration and slightly decreased recruitment pattern. Motor evoked potentials are abnormal with delayed central conduction time and normal motor responses from the cervical or lumbar roots. Sensory evoked potentials are normal. There is no treatment for this entity. Slow and mild degeneration of gait occurs over decades (Le Foretier et al 2001, Singer et al.2007).

Hereditary spastic paraplegia is a hereditary disorder of central motor tracts in the brain and spinal cord with no known etiology. It is characterized by retrograde axonal degeneration of the corticospinal tracts and posterior columns. It presents with spastic paraplegia with hyperreflexia and Babinski signs, with relative preservation of power which contrasts with other causes of spinal injury. The inheritance mode can be either autosomal dominant, autosomal recessive, or X-linked recessive. Each mode has its own genetic abnormality and clinical features. There is no treatment for such a condition (Salinas et al.2008).

\section{References}

[1] Aghakhani N, Parker F, David P, Lasjaunias P, Tadie M. Curable cause of paraplegia: spinal dural arteriovenous fistula. Stroke 2008; 39(10):2756-9.

[2] Anantharaju A, Baluch M, Van Thiel DH. Transverse myelitis occurring in association with primary biliary cirrhosis and Sjogren's syndrome. Digestive Disease Science. 2003; 48(4):830-3.

[3] Baron E, Young W. Cervical spondylotic myelopathy: a brief review of its pathophysiology, clinical course, and diagnosis. Neurosurgery 2007; 60(1):35-42.

[4] Bashir K, Whitaker JN. Importance of paraclinical and CSF studies in the diagnosis of MS in patients presenting with partial cervical transverse myelopathy and negative cranial MRI. Multilpe Sclerosis 2000; 6:312-6.

[5] Bay a, Oner AF, Etlik O, Yilmaz C, Caksen H. Myelopathy due to intrathecal chemotherapy: report of 6 cases. Journal of Pediatric Hematology Oncology 2005;27(5):270-2.

[6] Cheshire W, Santos C, Massey E, Howard J. Spinal cord infarction: Etiology and outcome. Neurology 1996; 47(2):321-331

[7] Clark AW, Cohen SR, Nissenblatt MJ, Wilson SK. Paraplegia following intrathecal chemotherapy: Neuropathological findings and elevation of myelin basic protein. Cancer 1982: 50:42-7.

[8] Cordonnier P, de Seze, Breteau G, Ferriby D, Michelin E, Stojkovic T, Pruvo JP, Vermersch P. Prospective study of patients presenting with acute partial transverse myelopathy. Journal of Neurology 2003; 250:1447-1452.

[9] Darnell RB, Posner JB. Paraneoplastic syndromes involving the nervous system. New England Journal of Medicine. 2003; 349:1543-1554.

[10]Darnell RB. Paraneoplastic neurological disorders. Archives of Neurology. 2004; 61:30-2.

[11]Darouiche R. Spinal epidural abscess. New England Journal of Medicine 2006; 355:2012-2020

[12] de Seze J, Lanctin C, Lebrun C, Malikova I, Papeix C, Wiertlewski S, Pelletier J, Gout O, Clerc C, Moreau C, Defer G, Edan G, Dubas F, Vermersch P. Idiopathic acute transverse myelitis: application of the recent diagnostic criteria. Neurology 2005; 65(12):1950-3.

[13]Di Rocco A. Diseases of the spinal cord in human immunodeficiency virus infection. Seminars in Neurology 1999;19(2):151-5. 
[14]Faig J, Busse O, Salbeck R. Vertebral body infarction as a confirmatory sign of spinal cord ischemic stroke: report of three cases and review of the literature. Stroke 1998; 29(1):239-243

[15]Fux CA, Pfister S, Nohl F, Zimmerli S. Cytomegalovirus-associated acute transverse myelitis in immunocompetent adults. Clinical Microbiology Infection. 2003; 9(12):1187-1190

[16]Harzheim M, Schlegel U, Urbach H, Klockgether T, Schmidt S. Discriminatory features of acute transverse myelitis: a retrospective analysis of 45 patients. Journal of Neurological Sciences. 2004; 217(2):217-223

[17]Helweg-Larsen S, Sorensen PS. Symptoms and signs in metastatic spinal cord compression: a study of progression from first symptom until diagnosis in 153 patients. European Journal of Cancer 1994; $30 \mathrm{~A}(3): 396-8$

[18]Hemmer B, Glocker FX, Schumacher M, Deuschl G, Lucking CH Subacute combined degeneration: clinical, electrophysiological, and magnetic resonance imaging findings. Journal of Neurology Neurosurgery Psychiatry 1998; 65:822-7

[19]Ishida T, Murayama S, Yamaguchi $K$, Urano $Y$, Iwata $M$. Arteriovenous malformation of the spinal cord. Virshows Archives Pathologic Anatomy 1989; 414:497-502.

[20]Kaplin A, Krishnan C, Deshpande D, Pardo c, Kerr D. Diagnosis and management of acute myelopathies. The Neurologist 2005; 11:2-18.

[21] Kincaid O, Lipton HL. Viral myelitis: an update. Current Neurology Neuroscience Repertoire. 2006; 6(6):469-474

[22]Koch C. Spinal dural arteriovenous fistula. Current Opinion in Neurology 2006; 19:69-75.

[23] Krishnan A, Halamgyi G. Acute transverse myelitis in SLE. Neurology 2004; 62(11):2087.

[24]Kumar N, Frohman EM. Spinal neurosarcoidosis mimicking an idiopathic inflammatory demyelinating syndrome. Archives of Neurology. 2004; 61(4):586-9.

[25] Le Foretier N, Maisonobe T, Piquard A, Rivaud S, Crevier-Buchman L, Salachas F, Pradat P, Lacomblez L, Meiningier V. Does primary lateral sclerosis exist? Brain 2001;124(10):1989-1999.

[26]Lee H, Im S, Kang M, Kim K, Kim S, Kim H, Kang J, Lee G. Irreversible paraplegia following one time prophylactic intrathecal chemotherapy in an adult patient with acute lymphoblastic leukemia. Yonsei Medical Journal 2008; 49(1):151-4.

[27]Mahfouz RA, Otrock ZK, Kanaan ZM, Sawaya RA, Taher AT. Homozygous methylenetetrahydrofolate $\mathrm{C} 677 \mathrm{~T}$ mutation in a woman with spinal cord infarction. European Journal of Neurology 2006; 13(9):e4.

[28]McCormick WE, Steinmetz MP, Benzel EC. Cervical spondylotic myelopathy: make the difficult diagnosis, and then refer for surgery. Cleveland Clinics Journal of Medicine. 2003; 70(10):899-904.

[29]Meurs L, Labeye D, Declercq I, Pieret F, Gille M. Acute transverse myelitis as a main manifestation of early stage II neuroborreliosis in two patients. European Neurology 2004; 52:186-8.

[30]Mok CC, Lau CS. Transverse myelopathy complicating mixed connective tissue disease. Clinical Neurology and Neurosurgery. 1995; 97(3):259-260

[31]Moser H, Mahmood A, Raymond G. X-linked adrenoleukodystrophy. Nature Clinical Practice Neurology 2007; 3(3):140-151.

[32]Pascal A, Coret F, Casanova B, Lainez M. Anterior lumbosacral polyradiculopathy after intrathecal administration of methotrexate. Journal of the Neurological Sciences 2008; 267:158-161.

[33] Orme HT, Smith AG, Nagel MA, Bert RJ, Mickelson TS, Gilden DH. VZV spinal cord infarction identified by diffusion-weighted MRI. Neurology 2007; 69(4):398-400.

[34] Saleh S, Saw C, Marzouk K, Sharma O. Sarcoidosis of the spinal cord: Literature review and report of eight cases. Journal of the National Medical Association 2006; 98(6):965-976.

[35]Salinas S, Proukakis C, and Crosby a, Warner T. Hereditary spastic paraplegia: clinical features and pathogenetic mechanisms. Lance Neurology 2008; 7:1127-1138.

[36] Sawaya R. Adrenoleukodystrophy: a review. The Neurologist 2000 6:214-9.

[37]Sevick RJ, Wallace CJ. MR imaging of neoplasms of the lumbar spine. Magnetic Resonance Imaging Clinics of North America 1999, 7(3):539-353.

[38] Shrivastava RK, Epstein FJ, Perin NI, Post KD, Jallo GI. Intramedullary spinal cord tumors in patients older than 50 years of age: management and outcome analysis. Journal of Neurosurgical Spine 2005; 2(3):249-255.

[39]Singer MA, Statland JM, Wolfe GI, Barohn RJ. Primary lateral sclerosis. Muscle Nerve 2007; 35:291-302

[40]Staudinger R; Henry K. Remission of HIV myelopathy after highly active antiretroviral therapy. Neurology 2000; 54(1):267-8.
[41] Toothaker T, Rubin M. Paraneoplastic neurological syndromes. The Neurologist 2009;15:21-33

[42] Torabi AM, Patel RK, Wolfe GI, Hughes CS, Mendelsohn DB, Trivedi JR. Transverse myelitis in systemic sclerosis. Archives of Neurology. 2004; 61(1):126-8. 